\title{
MedienPädagogik
}

$w w w$. medienpaed.com

Zeitschrift für

Theorie und Praxis

der Medienbildung

ISSN 1424-3636

Themenheft Nr. 18: Neue Medien und individuelle Leistungsdarstellung -

Möglichkeiten und Grenzen von ePortfolios und eAssessments

\section{Der Einsatz von E-Portfolios in der Berufsausbildung - Konzeption und Potenziale}

Uwe Elsholz und Sönke Knutzen

\begin{abstract}
In der beruflichen Bildung sind (E-)Portfolios zur Reflexion des Gelernten - anders als etwa an Hochschulen oder in allgemeinbildenden Schulen - in Theorie und Praxis gegenwärtig kaum existent. Konzepte und Studien zum Einsatz von E-Portfolios in der Berufsausbildung und der Weiterbildung fehlen weitgehend, so dass die Potenziale von Portfolioarbeit für eine selbstbewusstere und selbstbestimmtere Gestaltung beruflichen Lernens bisher ungenutzt bleiben.

Hier setzt dieser Beitrag an. Es wird ein Konzept vorgestellt, wie der Einsatz eines E-Portfolios die Berufsausbildung im dualen System unterstützen kann. Anhand des dargestellten Beispiels werden Möglichkeiten und Grenzen des Einsatzes von E-Portfolios in der beruflichen Bildung aufgezeigt.

Die Umsetzung des E-Portfolios findet im Rahmen des berufswissenschaftlich begründeten E-Learning-Konzepts der Kompetenzwerkst@tt statt (vgl. Howe/Knutzen 2007). Die Kompetenzwerkst@tt ist ein softwaregestütztes Ausbildungskonzept, das sich an realen beruflichen Arbeitsprozessen und Ansätzen des Situierten Lernens orientiert.Im Mittelpunkt des E-Portfolios als Teil der Kompetenzwerkst@ tt steht die lernortübergreifende Dokumentation und Reflexion der Ausbildungsinhalte der Dualen Berufsausbildung.

Im Beitrag wird gezeigt, wie die konzeptionellen Überlegungen zur Portfolioarbeit in der beruflichen Bildung vom Portfolio-Diskurs in anderen Bildungsbereichen beeinflusst sind, sich aber in der Umsetzung und in den konkreten Zielsetzungen deutlich unterscheiden. Es werden darüber hinaus weitere Möglichkeiten und Optionen von Portfolioarbeit in der beruflichen Bildung aufgezeigt.
\end{abstract}

\section{Ausgangslage: Portfolios in der beruflichen Bildung - bisher weitgehend ungenutzt}

Die Arbeit mit Portfolios findet gegenwärtig vor allem im Bereich allgemeinbildender Schulen grosse Aufmerksamkeit in Theorie und Unterrichtspraxis (vgl. Häcker 2005, 2007a; Brunner u. a. 2008; Endres u. a. 2008). Daneben gibt es vielfältige Ansätze im Bereich der Hochschulbildung, in denen E-Portfolios zum Einsatz kommen (vgl. Baumgartner u. a. 2009; Schaffert u. a. 2007; Hornung-Prähauser u. a. 2007). Im deutschsprachigen Raum findet sich zudem in der Lehrerbildung ein Schwerpunkt 
und in mehreren Bundesländern gibt es Bestrebungen, im Referendariat Portfolios einzuführen (vgl. Ness 2009; Häcker/Winter 2008; Gehler 2007).

In der beruflichen Bildung gibt es mit dem Berichtsheft, das obligatorisch von den Auszubildenden zu führen ist, eine Form der Dokumentation des Ausbildungsverlaufs, die jedoch weitgehend nur eine Kontrollfunktion besitzt. Es liegen bisher nur vereinzelte Erprobungsansätze zur Portfolioarbeit vor, die sich zudem weitgehend auf schulische Ausbildungsanteile beschränken (vgl. Brouer 2007; Schmidt 2005; Zeder 2006). Das nachfolgend vorgestellte Portfolio-Konzept im Rahmen des Projekts «Kompetenzwerkst@tt Elektrohandwerk»1 unterscheidet sich von den vorliegenden Ansätzen, da es nicht nur - wie etwa bei Zeder (2006) - auf ein einzelnes Fach in einer beruflichen Schule fokussiert ist, sondern die gesamte dreieinhalbjährige Berufsausbildung umfasst; zudem wird das Lernen an den unterschiedlichen Lernorten Betrieb, Überbetriebliche Ausbildungsstätte und Berufsschule in den Blick genommen. Ziel der Portfolioarbeit ist es, die Auszubildenden darin zu unterstützen, die in der Regel asynchron erworbenen praktischen Erfahrungen der betrieblichen Ausbildung und die eher fachtheoretischen Bezüge der berufsschulischen Ausbildung zusammenzutragen und zusammenzufügen.

Im Folgenden wird einführend die methodologische Anlage zur Entwicklung eines E-Portfolios in der Berufsausbildung geschildert, der ein zyklischer Prozess zwischen Theorie und Praxis zugrunde liegt. Dieser Struktur von theoretischen Bezügen und empirisch-praktischer Konzeption folgt auch der weitere Aufbau des Aufsatzes. Die theoretischen Bezüge werden vorgestellt und daran anknüpfend der gegenwärtige Entwicklungsstand des E-Portfolios der Kompetenzwerkst@tt Elektrohandwerk aufgezeigt. Die leitenden Forschungsfragen spannen schliesslich den Bogen für die formative Evaluation des Einsatzes des E-Portfolios in der Berufsbildungspraxis. Über die konkreten Projektarbeit hinausgehend werden abschliessend weitere Möglichkeiten von Portfolioarbeit in der beruflichen Aus- und Weiterbildung erörtert.

\section{Methodisches Vorgehen zur Entwicklung und Erprobung eines E-Portfolios in der Berufsausbildung}

Die Entwicklung und Erprobung des nachfolgend vorgestellten E-Portfolios folgt einem zyklischen Vorgehen, bei dem sich theoretische Bezüge und praktische Umsetzungsschritte wechselseitig ablösen und einander ergänzen. Mit diesem zyklischen Vorgehen zwischen Theorie und Praxis soll eine doppelte Anschlussfähigkeit

1 Das Projekt «Kompetenzwerkst@tt Elektrohandwerk» entwickelt für den Ausbildungsberuf des Elektronikers der Fachrichtung Energie- und Gebäudetechnik ein softwaregestütztes Ausbildungskonzept. Es wird federführend vom Institut für Technik, Arbeitsprozesse und Berufliche Bildung der TU Hamburg-Harburg und dem Institut Technik und Bildung der Universität Bremen mit Beteiligten des Elektrohandwerks in den Regionen Hamburg, Dresden und Bremen durchgeführt. Die Laufzeit des vom Bundesministerium für Bildung und Forschung (BMBF) und dem Europäischen Sozialfonds (ESF) geförderten Projekts ist vom 1.5.2008-30.4.2011. (www.kompetenzwerkstatt.net) 
sowohl an die Berufsbildungspraxis als auch an die wissenschaftliche Diskussion gewährleistet werden (vgl. Moser 1995, S. 73; Elsholz 2006, S. 97 ff.). Der Anspruch, Theoriebildung und Praxisinnovation zu generieren, entspricht einem in der Berufsbildungsforschung verbreiteten Ansatz, der als «responsive Forschung» (Euler 2007, S. 88) gekennzeichnet werden kann. «Responsive Forschung verbindet Erkenntnisgewinnung mit Praxisgestaltung im Rahmen der Entwicklung, Erprobung und Evaluation von Innovationsprojekten» (ebd.).

Auf theoretischer Ebene wird bei der Entwicklung des E-Portfolios an das E-Learning-Konzept der Kompetenzwerkst@tt und seinen lerntheoretischen Bezügen angeknüpft (vgl. Howe/Knutzen 2007). Darüber hinaus werden bisher vorliegende Ausarbeitungen und Erkenntnisse zu (E-)Portfolios sowohl aus dem Bereich der allgemeinbildenden Schulen als auch aus Einsatzszenarien an Hochschulen für die berufliche Bildung fruchtbar gemacht. Schliesslich werden Fragen aus der Berufsbildungsforschung bei der Bearbeitung aufgenommen.

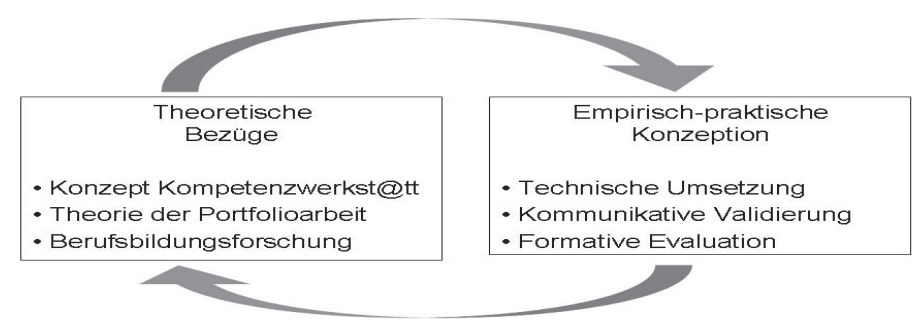

Abb. 1: Zyklischer Prozess der Entwicklung und Erprobung des E-Portfolios

Seitens der empirisch-praktischen Konzeption wird zum einen die technische Umsetzung der konzeptionellen Ansprüche an das E-Portfolio bearbeitet. Darüber hinaus werden über die Präsentation der Konzeption des E-Portfolios sowie von Zwischenergebnissen vielfach Rückmeldungen aus der Berufsbildungspraxis in einem methodisch kontrollierten Prozess aufgenommen. Diese mehrfache kommunikative Validierung ist integraler Bestandteil der responsiven Forschung. In der weiteren Erprobung und Umsetzung erfolgt eine formative Evaluation, die sich an leitenden Fragestellungen orientiert und die sowohl der Erkenntnisgewinnung als auch der verbesserten Praxisgestaltung dient.

Zudem erfolgt ein permanenter Abgleich von neueren theoretischen Entwicklungen mit der empirisch-praktischen Konzeption, so dass sich ein zyklischer Entwicklungs- und Forschungsprozess ergibt. 


\section{Konzeption und lerntheoretische Verortung der Kompetenzwerkst@tt}

Die Konzeption und Umsetzung des E-Portfolios erfolgt im Rahmen des Projekts «Kompetenzwerkst@tt Elektrohandwerk», das die Erstellung einer Lernsoftware für Elektroniker der Fachrichtung Gebäude- und Systemtechnik zum Ziel hat. Die Lernsoftware nimmt das berufswissenschaftlich begründete E-Learning-Konzept der Kompetenzwerkst@tt (vgl. Howe/Knutzen 2007) auf und erweitert es durch die Integration eines E-Portfolios.

Die Kompetenzwerkst@tt ist ein umfassendes Lehr-Lernkonzept, das sich im gesamten Bereich der gewerblich-technischen Berufsbildung einsetzen lässt. Im Unterschied zu sog. "Stand-Alone-Lösungen» wie z.B. die klassische Sprachlernsoftware, die für definierte Lernzwecke programmiert sind und u. a. die Person des Lehrenden substituieren, kommt Lehrern und Ausbildern in der Kompetenzwerkst@tt eine Schlüsselfunktion zu. Die Lehrenden sind für die Umsetzung der Ausbildung verantwortlich und übernehmen eine beratende und unterstützende Rolle. Lernwege sind nicht vorgegeben, sondern ergeben sich erst aus dem jeweiligen Aufgabenkontext.

Das E-Learning-Konzept der Kompetenzwerkst@tt nimmt Diskussionen in der beruflichen Bildung auf, die sich von einer stark fachsystematischen Ausrichtung abgrenzen. Sie orientiert sich im Aufbau an realen Arbeitsprozessen und lerntheoretisch an Ansätzen der Situierten Kognition (Lave/Wenger 1991; Cognition and Technology Group 1990; DeWitt/Czerwionka 2007, S. 60 ff.). Danach wird Lernen als aktiver individueller Konstruktionsprozess verstanden und vollzieht sich in Verbindung mit bereits bestehendem Vorwissen und -erfahrungen. Lernanlass sollte eine interessante und authentische Problem- bzw. Aufgabenstellung sein (vgl. Mandl u. a. 2002). In der Kompetenzwerkst@tt erschliesst sich der Lernende die Lerninhalte über Lern- und Arbeitsaufgaben - ein in der beruflichen Bildung verbreiteter didaktischer Ansatz (vgl. Howe/Berben 2005). Die Lern- und Arbeitsaufgaben sind in berufsbezogene Situation an den unterschiedlichen Lernorten eingebettet.

Die Erstellung der Lernsoftware im Rahmen des Projekts «Kompetenzwerkst@tt Elektrohandwerk» orientiert sich zusätzlich an Prinzipien des Rapid-E-Learning (vgl. Payome 2008; Knutzen/Howe 2009). Sie erfolgt daher mit Hilfe von PowerPoint, so dass vor allem Ausbilder und Berufsschullehrer individuelle Anpassungen vornehmen können, um eigene Lern- und Arbeitsaufgaben in der Lernsoftware zu verankern. Die kostenfreie Software wird den Auszubildenden auf einem USB-Stick zur Verfügung gestellt, so dass jeder Auszubildende eine eigene individualisierbare Lernsoftware besitzt.

Die Kompetenzwerkst@tt Elektrohandwerk besteht aus zwölf Handlungsfeldern, die den Beruf des Elektronikers widerspiegeln. Die Struktur der lernortübergreifenden Handlungsfelder wurde in einem mehrstufigen Verfahren auf Grundlage der vorhandenen Ordnungsmittel und unter Einbeziehung von Berufsbildungspraktikern entwickelt (vgl. Elsholz/Knutzen 2009). 


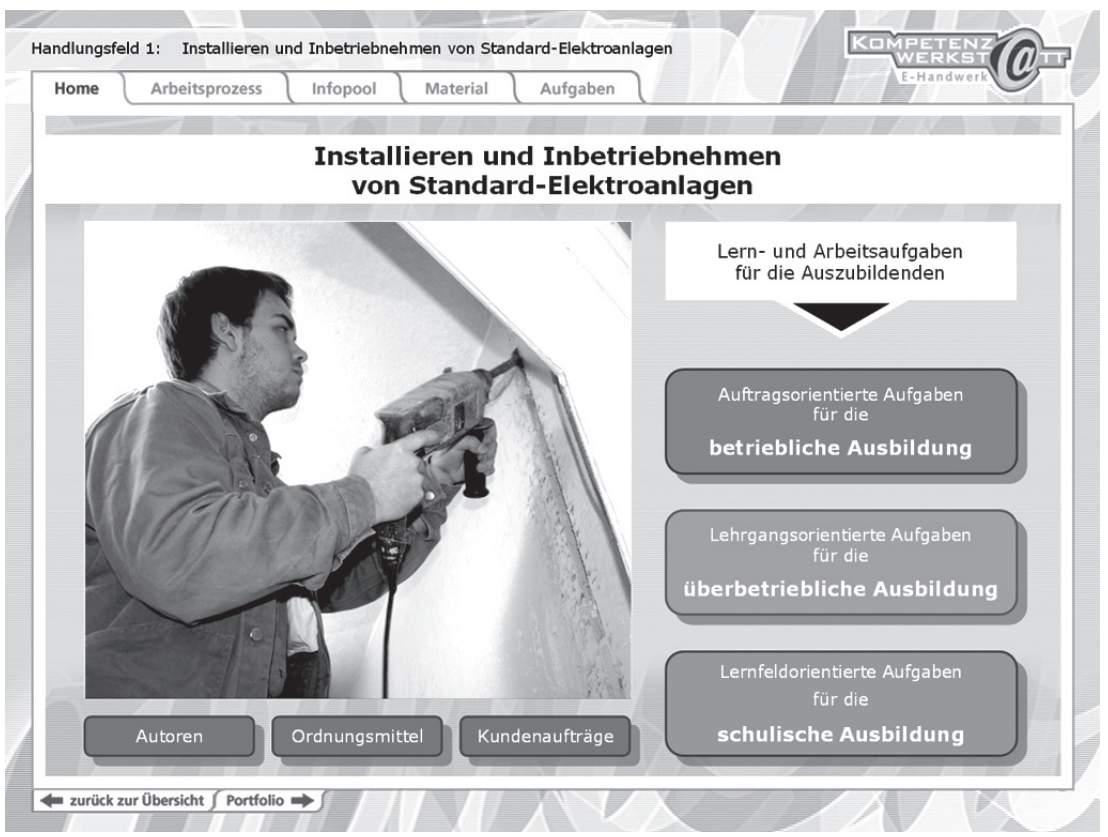

Abb.2: Einstiegsseite in ein Handlungsfeld der Lernsoftware

\section{Ziele des E-Portfolios in der Kompetenzwerkstatt}

Die Lernsoftware Kompetenzwerkst@tt wird nun durch ein E-Portfolio erweitert. Dabei wurde hinsichtlich der Konzeption des E-Portfolios neben dem berufbildungsspezifischen Bezugsrahmen vor allem auf Arbeiten von Thomas Häcker Bezug genommen, der sich eingehend mit der Entwicklung von Portfolioarbeit im Bereich allgemeinbildender Schulen auseinander gesetzt hat. Zwei Grundideen sind entsprechend für die Umsetzung im Rahmen des Projekts «Kompetenzwerkst@tt Elektrohandwerk» leitend:

«Portfolios dienen der mehr oder weniger selbstbestimmten Darstellung des eigenen Könnens (Kompetenzdarstellung) und der eigene Entwicklung anhand (selbst) ausgewählter Leistungsprodukte» (Häcker 2008, S. 34; Hervorhebung durch Verf.). «Gezielte Reflexionen über das eigene Lernen sind das Herzstück des Portfolioprozesses» (Häcker 2005, S. 6; Hervorhebung durch Verf.).

Aus diesen grundsätzlichen Orientierungen haben sich folgende Ziele für den Einsatz und die Struktur des E-Portfolios in der Lernsoftware ergeben:

- Dokumentation des Lern- und Ausbildungsprozesses als elektronisches Berichtsheft

Als ein erstes wesentliches Ziel soll mit dem E-Portfolio der eigene Lernprozess im Rahmen der Ausbildung festgehalten werden. Daher wird eine prozessbe- 
gleitende Dokumentation des Arbeitens und Lernens an den unterschiedlichen Lernorten über den gesamten Verlauf der Ausbildung vorgenommen. Alle Tätigkeiten im Betrieb, Unterrichtssequenzen in der Berufschule und Lehrgangsinhalte in der überbetrieblichen Bildungsstätte werden im E-Portfolio festgehalten. Mit dieser Dokumentation des Ausbildungsverlaufs soll mittelfristig das obligatorisch zu führende das Berichtsheft ersetzt werden, das Voraussetzung für die Zulassung zur Abschlussprüfung ist.

In dieser engen Kopplung des Ausbildungsportfolios an das Berichtsheft wird von den Berufsbildungspraktikern das wesentliche motivationale Moment gesehen, das E-Portfolio tatsächlich zu führen, da der Nutzen der Tätigkeit offenbar wird. Dieser transparente Nutzen und die Wichtigkeit der Motivation als Voraussetzung für die erfolgreiche Einführung eines solchen Instruments heben verschiedene Autoren hervor (vgl. Brouer 2007, S. 42; Zeder 2006).

- Reflexion über Ausbildungsinhalte verbessern

In Erweiterung der chronologischen Dokumentation des Ausbildungsverlaufs ermöglicht das Portfolio auch eine inhaltlich orientierte Reflexion. Es stellt sich für eine qualitative hochwertige Ausbildung und zur Entwicklung beruflicher Professionalität die Herausforderung, wie das an den unterschiedlichen Lernorten in der Berufsausbildung Gelernte miteinander in Verbindung gebracht werden kann. Die fehlende Verzahnung von Theorie und Praxis stellt sich für Ausbildende als grosses Problem dar, da verwandte Themen in der Schule und im Betrieb in der Regel zu sehr unterschiedlichen Ausbildungszeitpunkten vorkommen bzw. behandelt werden und daher häufig nicht in Beziehung zueinander gesetzt werden können. Die mangelnde Kooperation der Lernorte Betrieb und Berufsschule ist in der beruflichen Bildung ein klassisches Thema, das sich seit Jahrzehnten in wissenschaftlichen und berufsbildungspolitischen Diskussionen findet (vgl. u. a. Euler 2003). Gelungene Beispiele von langfristiger und erfolgreicher Kooperation zwischen den Lernorten finden sich äusserst selten. Die strukturellen Probleme der institutionalisierten Kooperation und unterschiedliche Logiken von Betrieben und Berufsschulen stehen dem im Wege. Daher wird mit dem E-Portfolio die Lernortkooperation nicht institutionell verstanden als Kooperation von Schule und Betrieb, sondern es wird eine individuelle «Lernortkooperation im Kopf» unterstützt (vgl. Elsholz/Knutzen 2009). Mit dem Ansatz der Portfolioarbeit wird angestrebt, eine solche «Lernortkooperation im Kopf» zu ermöglichen, indem der Auszubildende die unterschiedlichen Aspekte, die zu einem beruflichen Handlungsfeld gehören, in seinem Portfolio zusammenfasst. Damit sollen Bezüge von berufsschulischen zu betrieblichen Ausbildungsanteilen besser hergestellt werden können, als dies bisher der Fall war. 
- Reflexion über den Ausbildungsverlauf und die berufliche Entwicklung verbessern

Das E-Portfolio in der Kompetenzwerkst@tt Elektrohandwerk ist so aufgebaut, dass sich die Struktur an den relevanten beruflichen Handlungsfeldern, also den zentralen Tätigkeitsclustern (vgl. Bader 2000, Howe/Knutzen 2007) des Elektronikers orientiert. Über das Führen des E-Portfolios verbessert sich damit die Transparenz über die Ausbildungsinhalte und es können Lücken in der Ausbildung erkannt werden. Somit erweitern sich auch die Möglichkeiten des Ausbildenden, selbst zu einer besseren Ausbildungsqualität beizutragen und fehlende Ausbildungsbestandteile an den unterschiedlichen Lernorten einzufordern. Dieser Prozess der stärkeren Mitgestaltung der Lern- und Kompetenzentwicklungsprozesse bedarf allerdings der Unterstützung, wobei erst die formative Evaluation aufzeigen wird, inwieweit diese Unterstützung etwa durch Tutorials oder durch Lehrende zu leisten ist.

\section{Umsetzung des E-Portfolios}

Den geschilderten Zielsetzungen folgend, ist das E-Portfolio auf chronologische Dokumentation und inhaltliche Reflexion des Ausbildungsverlaufs ausgerichtet. Ebenso wie die gesamte Lernsoftware Kompetenzwerkst@tt befindet sich das EPortfolio auf einem USB-Stick, der im Besitz des Auszubildenden ist. Die Verfügungsgewalt über das Portfolio befindet sich damit ausschliesslich beim Auszubildenden, also dem Ersteller des Portfolios. Das Vorgehen zum Führen des Portfolios ist wie folgt angelegt:

Im E-Portfolio werden alle Tätigkeiten in der Ausbildung festgehalten und reflektiert. Vier Leitfragen unterstützen den Auszubildenden in einer Eingabemaske dabei. Die Fragen unterscheiden sich je nach Lernort in der Wortwahl, folgen aber im Betrieb, der Berufsschule und der Überbetrieblichen Ausbildungsstätte (ÜBA) der gleichen Struktur. Die ersten beiden Fragen nach dem Inhalt der Tätigkeit («Wie lautete der Auftrag?»; «Was war dabei meine Aufgabe?») dienen vornehmlich der Dokumentation des Ausbildungsverlaufs. Alle Tätigkeiten im Betrieb, Unterrichtssequenzen in der Berufschule und Lehrgangsinhalte in der überbetrieblichen Ausbildungsstätte werden damit festgehalten. Die Antworten auf diese Fragen werden für die Erstellung des Berichtshefts genutzt. Das E-Portfolio ermöglicht einen chronologischen Ausdruck der Eingaben zu diesen Fragen als Pdf-Dokument, welches den formalen Vorgaben eines Berichtshefts entspricht. Dieser Teil des Portfolios kann in ausgedruckter Form dem Ausbilder vorgelegt werden und wird damit für Dritte sichtbar.

Zwei weitere Fragen («Was habe ich Neues gelernt oder erfahren?»; «Wo gab es Schwierigkeiten und Probleme?») dienen stärker der Reflexion der Ausbildungsinhalte. Diese Fragen zielen auf die persönliche Reflexion und sind nur für den Nutzer des Portfolios sichtbar. 


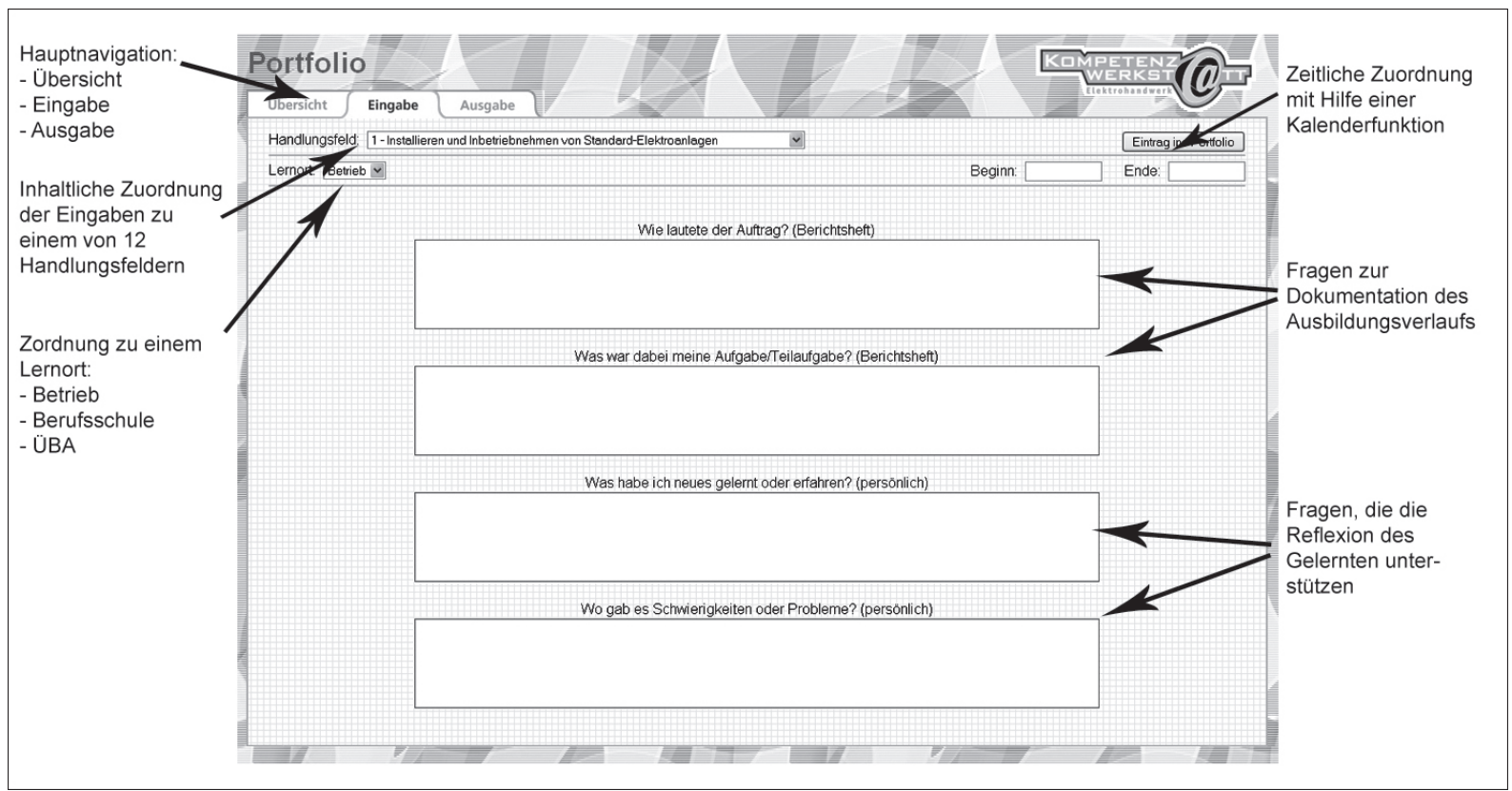

Abb. 3: Eingabemaske im Ausbildungsportfolio

Die einzelnen Einträge werden vom Auszubildenden jeweils einem beruflichen Handlungsfeld zugeordnet. Diese Zuordnung einzelner Arbeitsaufträge und schulischer Lernsituationen ist bereits ein wichtiger Teil der Reflexion und soll die berufliche Identitätsbildung unterstützen. Die lernortübergreifenden Handlungsfelder bilden das verbindende Element der Lernsoftware Kompetenzwerkst@tt und des E-Portfolios. Das E-Portfolio nimmt diese Struktur auf und stellt sie in der Startseite dar. Daneben sind die Lernorte Betrieb (und Überbetriebliche Ausbildungsstätte ÜBA, die rechtlich zum Lernort Betrieb zählt) und Schule zu sehen und es wird angezeigt, ob und wie viele Einträge bereits vorhanden sind (siehe Abb. 4).

Hinsichtlich der technischen Umsetzung der konzeptionellen Vorstellungen hat sich gezeigt, dass vorhandene Ansätze und Software für E-Portfolios die Anforderungen nicht erfüllen. Insbesondere die Umsetzung und Lauffähigkeit auf einem USB-Stick hat eine Eigenentwicklung erzwungen. Daher erfolgt die technische Umsetzung des E-Portfolios mit einem virtuellen Server (XAMPP), mySQL und php. Mit dieser Lösung kann der Nutzer sein persönliches Portfolio jederzeit und an unterschiedlichen Orten (z.T. in der Berufsschule, im Betrieb oder zu Hause) auf seinem eigenen USB-Stick führen. 


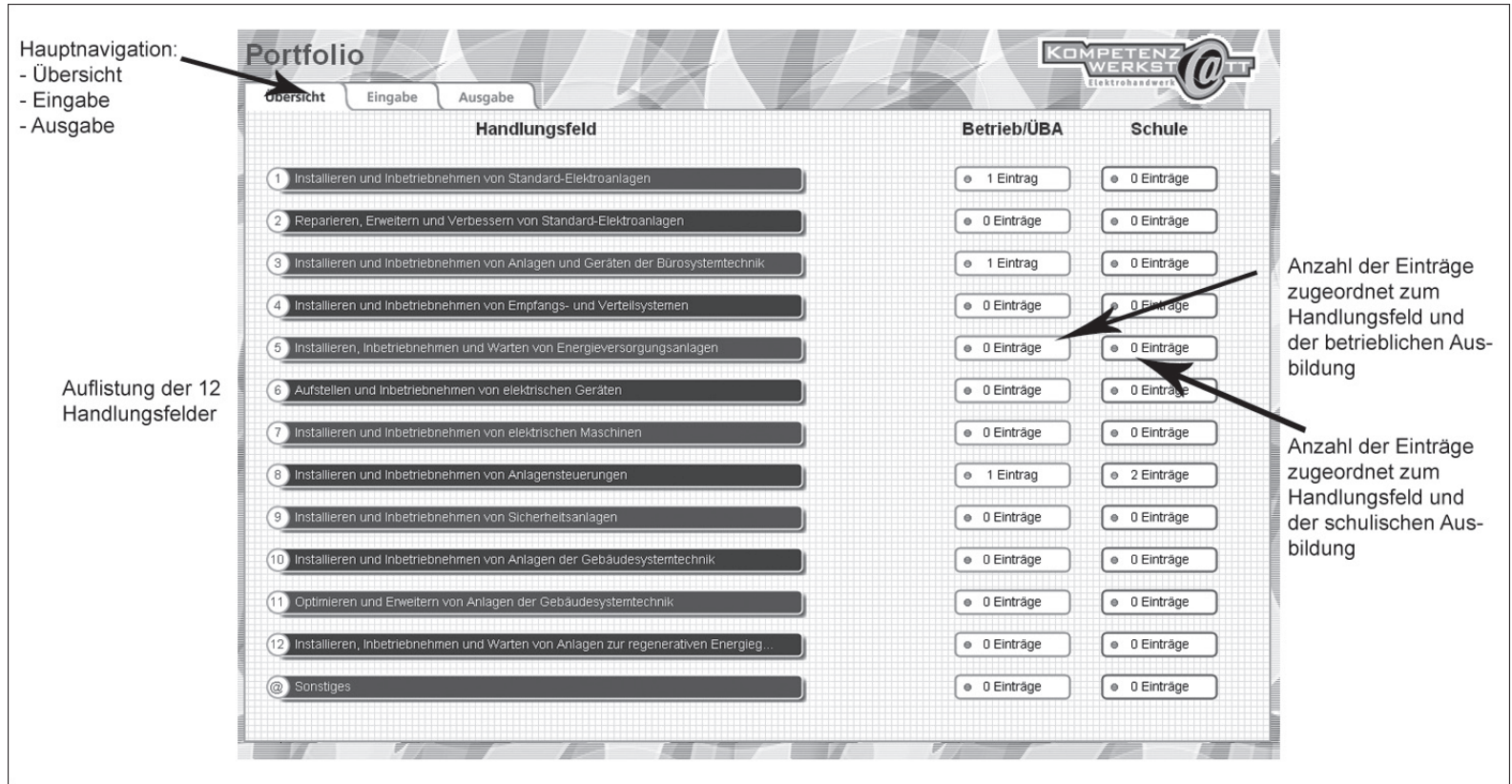

Abb. 4: Einstiegsseite des Ausbildungsportfolios

\section{Formative Evaluation}

Ein wichtiger Aspekt bei der Entwicklung und konkreten Ausgestaltung des E-Portfolios in der Kompetenzwerkst@tt ist im Sinne des responsiven Forschungsansatzes die kommunikative Validierung mit den Berufsbildungspraktikern. Es wird nicht eine abgeschlossene theoretische Konzeption umgesetzt, sondern es wurden und werden Zwischenergebnisse vorgestellt und diese mit Ausbildern und Berufsschullehrern diskutiert. Durch dieses Vorgehen konnten bereits wichtige Aspekte zur konkreten Gestaltung als auch zum Einsatz des E-Portfolios in der Berufsbildungspraxis in die Entwicklungsarbeiten einbezogen werden.

Weitergehend als die kommunikative Validierung erfolgt eine formative Evaluation zum Einsatz des E-Portfolios. Dabei stehen folgende leitenden Fragen im Fokus, die sich sowohl auf den Diskurs zur Portfolioarbeit beziehen als auch für die praktische Umsetzung von Relevanz sind:

- Wie stark sollte das Portfolio strukturiert sein durch Leitfragen und Hilfestellungen?

Vorliegende empirische Untersuchungen zeigen, dass eine Strukturierung von Portfolios über bestimmte Fragestellungen grundsätzlich hilfreich ist (vgl. Hübner u. a. 2007). Es geht bei diesem Aspekt zum einen darum, die angemessene Art und Anzahl der Fragen im Portfolio zu entwickeln, damit das E-Portfolio handhabbar bleibt. Dabei ist zum einen die Zielgruppe (Auszubildende im Elektrohandwerk) bzw. deren kognitive Voraussetzungen zu berücksichtigen, 
zum anderen sind aber auch die Rahmenbedingungen des Einsatzes in der Berufsbildungspraxis an den unterschiedlichen Lernorten zu bedenken (z. B. bezogen auf Zeiten und technische Voraussetzungen zum Führen des E-Portfolios). Darüber hinaus gilt es auch zu fragen, welche weiteren Hilfestellungen (z.B. Tutorials) den erfolgreichen Einsatz des E-Portfolios unterstützen können.

- Wie kann mit dem Portfolio Reflexion über den Ausbildungsverlauf angeregt werden?

Durch das E-Portfolio soll die Verbindung von Theorie und Praxis unterstützt und eine «Lernortkooperation im Kopf» ermöglicht werden. Das Thema Reflexion bzw. Unterstützung von Reflexion wird in der Portfolioarbeit noch weitgehend ohne theoretisches Konzept bearbeitet und es gibt wenig empirische Erkenntnisse dazu (vgl. Czerwionka/Knutzen/Bieler in diesem Band). In diesem Zusammenhang geht es darum zu fragen, welche Rolle das Portfolio dabei spielen kann, eine solche Verbindung von Theorie und Praxis zu unterstützen.

- Welche äusseren Rahmenbedingungen fördern oder behindern den Einsatz von E-Portfolios in der Ausbildung?

Die vorliegenden Befunde zur Portfolioarbeit beziehen sich vorrangig auf die konkrete Gestaltung von Portfolios. Ob und wie Portfolios aber tatsächlich eingesetzt werden (können), hängt - zumindest in der beruflichen Bildung - sehr stark von den unterschiedlichen Rahmenbedingungen ab. Hier gilt es, fördernde und hemmende Faktoren des Einsatzes zu identifizieren. Wie Gillen (2006) für das verwandte Thema Kompetenzanalysen gezeigt hat, wirken die Rahmenbedingungen des Einsatzes auch darauf zurück, inwiefern solche Verfahren kompetenzförderlich wirken.

Entsprechend den Zielsetzungen der formativen Evaluation werden sowohl die Nutzer (Auszubildende) als auch Ausbilder und Berufsschullehrer befragt. Während zur Befragung der Auszubildenden in erster Linie quantitative Befragungen geplant sind, sollen Ausbilder und Berufsschullehrer in Leitfadeninterviews befragt werden. Die Evaluation beginnt mit der Erprobung des Referenzmoduls der Lernsoftware im Herbst 2009 und ist zunächst bis Anfang 2011 geplant. In diesem Prozess erfolgt auch eine weitere Ausdifferenzierung der leitenden Fragestellungen und des methodischen Vorgehens.

\section{Potenziale von E-Portfolios in der beruflichen Bildung}

Über die im Rahmen des beschriebenen Projekts verfolgten Zielsetzungen und Fragestellungen deuten sich für die berufliche Bildung weitere Optionen und Potenziale von Portfolioarbeit an. Im Anschluss an die geschilderten Projektarbeiten wird bei erfolgreicher Entwicklung und Erprobung eine Weiterentwicklung ange- 
strebt. Diese Weiterentwicklung kann an folgenden Punkten ansetzen, wobei sich die geschilderten Potenziale auch im Rahmen anderer Projekte und Ansätze erschliessen lassen.

- Ausbildungsportfolio als Grundlage für Bewerbungen

Ein Ausbildungsportfolio kann als Grundlage für Bewerbungen im Anschluss an die Ausbildung dienen. Dabei soll durch die Auswahl bestimmter Teilbereiche und deren adäquate Präsentation aus dem bisherigen Ausbildungsportfolio ein Bewerbungsportfolio werden. Entsprechend dem zyklischen Vorgehen bei der Entwicklung und Erprobung des Portfolios kann dieses Ziel in die weitere Arbeit einfliessen, sofern ausreichend Ressourcen vorhanden sind. In der praktischen Umsetzung muss dann für diese Funktion eine entsprechende Darstellung gefunden werden. Dabei ist zu berücksichtigen, dass die Auswahl aus dem vorhandenem Material des Portfolios durch den Auszubildenden selbst zu erfolgen hat (vgl. Häcker 2007b).

- Portfolio als Grundlage weiterer Berufswegeplanung/Weiterbildung

Das E-Portfolio kann über die Ausbildung hinaus als Grundlage einer weiteren Berufswegeplanung sowie zur Planung der eigenen Weiterbildung dienen. Über die Reflexion des in der Ausbildung Gelernten können eigene Stärken und Interessen besser erkannt und damit auch weiter entwickelt werden. Hier sind Verbindungen zu Fragen der Kompetenzanalyse und Kompetenzbilanzierung zu ziehen und zu schaffen, um das Portfolio entsprechend zu gestalten (vgl. Gillen 2006; Ness 2009). Dabei ist zu vermuten, dass ein solcher Prozess zusätzlicher Beratung bedarf, wobei ein entsprechendes Portfolio eine gute Grundlage für Beratungsprozesse sein kann.

- Portfolios als alternative Form der Leistungsbeurteilung

Die Entwicklung und Verbreitung von Portfolioarbeit war in amerikanischen allgemeinbildenden Schulen eng verbunden mit der Suche nach alternativen Formen der Leistungsbeurteilung (vgl. Häcker 2007a). Entsprechend ist zu erwägen, wie Portfolioarbeit auch in der beruflichen Bildung als eine Form der alternativen Leistungsbeurteilung eingesetzt werden kann. Neben der Berufsschule bieten sich gerade in der beruflichen Bildung die Abschlussprüfungen. Diese werden bereits heute in unterschiedlicher Form und mit unterschiedlichen Elementen durchgeführt. Ein Portfolio als Teil einer Abschlussprüfung oder als vorbereitendes Element zur Abschlussprüfung böte hier Möglichkeiten, weniger punktuelle Leistungen zu bewerten, sondern den gesamten Ausbildungsverlauf in den Blick zu nehmen. Für die Weiterbildung deutet Schmidt (2005) an, wie bezogen auf das IT-Weiterbildungssystem Portfolios zur Leistungsbeurteilung hilfreich sein können. 
- Arbeiten und Lernen in der Weiterbildung verbinden

Die bereits oben durch die Einbeziehung unterschiedlicher Lernorte angedeutete Verbindung von Arbeiten und Lernen im Rahmen von Portfolioarbeit gewinnt besonders bezogen auf die Weiterbildung zusätzliche Relevanz. In der beruflichen Bildung hat das Lernen im Prozess der Arbeit in den letzten zwei Jahrzehnten grosse Aufmerksamkeit erfahren (vgl. z. B. Dehnbostel u. a. 2007). Arbeitsnahes Lernen liesse sich mit Hilfe eines Portfolios dokumentieren und reflektieren, wie auch Schmidt (2005) herausstellt: «Berufspädagogisch entspricht damit die Methode des Portfolio in wesentlichen Aspekten dem, was für ein modernes arbeitsplatzbezogenes Lernen bedeutsam ist: nicht nur Arbeitsprozesse vollziehen zu können, sondern sich auch Rechenschaft darüber zu geben, wie gelernt wurde und wie das erarbeitete Know-how auf diese Weise auch in andere Zusammenhänge hinein übertragen werden kann» (ebd., Abs. 38). Hier deuten sich weitere Potenziale von Portfolioarbeit für die Weiterbildung an. In Erweiterung von Arbeiten zur Entwicklung und Gestaltung arbeitsintegrierter Lernformen (vgl. Elsholz/Pross 2008) wird dieser Aspekt gegenwärtig in einem weiteren Projekt bearbeitet, in dem eine Kompetenzwerkst@tt für die berufliche Weiterbildung in der Abfallverbrennung entwickelt wird.

\section{Grenzen von Portfolioarbeit}

Die Grenzen des Einsatzes von E-Portfolios liegen - das zeigen erste Ergebnisse im Rahmen der kommunikativen Validierung mit Berufsbildungspraktikern - besonders in den strukturellen Bedingungen der beruflichen Bildung. Zu unterscheiden sind dabei Begrenzung hinsichtlich des bestehenden rechtlichen Rahmens (z. B. Prüfungsanforderungen) und andererseits Einschränkungen durch die gegenwärtige Berufsbildungspraxis in Berufsschulen und Betrieben. Diese lässt sich nur punktuell verändern und unterliegt unterschiedlichen Restriktionen (z. B. zeitlich, räumlich und technisch).

Im Rahmen des Projekts stellt sich die Herausforderung, dass E-Portfolio anschlussfähig an die Praxis der Ausbildung zu gestalten. Um das Führen des Portfolios als Berichtsheft zu ermöglichen, mussten hinsichtlich der konkreten Gestaltung des E-Portfolios Kompromisse eingegangen werden, z. B. um detaillierte Vorgaben zur Form des Berichtshefts zu berücksichtigen. Doch nur, wenn die Praxisbedingungen ausreichend berücksichtigt werden und die Auszubildenden zum Führen des Portfolios zu motivieren sind, besteht die Chance, dass das E-Portfolio auch zum Einsatz kommt. Diese Bedingungen genauer zu analysieren ist Teil des Evaluationsprozesses.

Auch für die weiterhin dargestellten Potenziale gilt es, Anschlussfähigkeit an die Berufsbildungspraxis herzustellen. Im Rahmen von Projekten und Modellversuchen sind dabei die Praxis als auch rechtliche Grenzen zu erweitern (z. B. veränderte Führung von Berichtsheften, neue Prüfungsformen, Portfolio als Unterlage 
zur Prüfungsvorbereitung), um die verschiedenen Potenziale von Portfolioarbeit nutzen zu können.

\section{Fazit und Ausblick}

In diesem Beitrag wurde sowohl eine konkrete Konzeption und Umsetzung eines E-Portfolios in der Berufsausbildung dargestellt, als auch darüber hinausgehend Potenziale von Portfolioarbeit für die aufgezeigt, die über den beschriebenen Ansatz hinausweisen und auch die berufliche Weiterbildung betreffen. An dieser Stelle soll zusammenfassend noch einmal auf folgende übergreifende Aspekte aufmerksam gemacht werden:

Für die berufliche Bildung stellen sich die Einsatzmöglichkeiten und die Zielsetzungen zum Portfolioeinsatz in spezifischer Weise dar. Befunde und Konzepte aus anderen Bildungsbereichen sind dabei zu berücksichtigen und hilfreich - doch überwiegen bildungsbereichsspezifische Herausforderungen und Fragestellungen.

Neben vielfältigen Aspekten der konkreten Ausgestaltung eines Portfolios ist deutlich geworden, dass die Rahmenbedingungen des Einsatzes eine zentrale Rolle bei der Betrachtung von Portfolioarbeit spielen müssen. Sie wirken zum einen auf die konkrete Gestaltung zurück und sind zum anderen wesentlich dafür verantwortlich, ob und in welcher Form ein entwickeltes Portfolio eingesetzt wird.

Schliesslich ist - darauf weist Häcker verstärkt hin - bei der Entwicklung und dem Einsatz von Portfolios die Haltung und Einstellung der Entwickler und der Anwender ganz entscheidend (vgl. Häcker 2007b). Das im Rahmen der Kompetenzwerkst@tt entwickelte E-Portfolio soll langfristig zur Stärkung der Selbststeuerung und damit zur «berufsbiografischen Gestaltungskompetenz» (Hendrich 2004) beitragen.

Abschliessend ist zu festzuhalten, dass mit der Portfolioarbeit in der beruflichen Bildung zwar vielfältige Ziele und Potenziale verbunden sind, dass aber die theoretischen und praktischen Arbeiten dazu bisher kaum entwickelt sind. Insofern ist in diesem Bereich ein grosser Bedarf sowohl an Entwicklungs- als auch an Forschungsarbeit zu konstatieren.

\section{Literatur}

Bader, Reinhard. «Konstruieren von Lernfeldern - Eine Handreichung für Rahmenlehrplanausschüsse und Bildungsgangkonferenzen in technischen Berufsfeldern.» Lernen in Lernfeldern. Theoretische Analysen und Gestaltungsansätze zum Lernfeldkonzept. Hrsg. v. Reinhard Bader und Peter F. Sloane. Paderborn: Eusl, 2000. 33-50.

Baumgartner, Peter; Himpsl, Klaus; Zauchner, Sabine. Einsatz von E-Portfolios an (österreichischen) Hochschulen: Zusammenfassung. (2009): www.peter. baumgartner.name/publications-de/e-portfolio-bericht-zusammenfassung.pdf (18.06.2009). 
Brouer, Birgit. "Pädagogische Portfolios in der Diplomhandelslehrausbildung.» Portfolio - Kompetenzen - Standards. Neue Wege in der Lehrerbildung für berufsbildende Schulen. Hrsg. v. Eva M. Hertle und Peter F. Sloane. Paderborn: Eusl, 2007. 31-44.

Brunner, Ilse; Häcker, Thomas; Winter, Felix (Hrsg.). Das Handbuch Portfolioarbeit. 2. Aufl. Seelze-Velber: Kallmeyer, 2008.

Cognition and Technology Group at Vanderbilt. «Anchored instruction and its relationship to situated cognition.» Educational Researcher 19.3 (1990): 2-10.

Czerwionka Thomas; Knutzen, Sönke; Bieler, Detlev. «Mit ePortfolios selbstgesteuert lernen - Ein Ansatz zur Reflexionsförderung im Rahmen eines hochschulweiten ePortfoliosystems.» MedienPädagogik 18 (26.3.2010): www.medienpaed.com/18/czerwionka1003.pdf.

Dehnbostel, Peter; Elsholz, Uwe; Gillen, Julia (Hrsg.). Kompetenzerwerb in der Arbeit. Perspektiven arbeitnehmerorientierter Weiterbildung. Berlin: edition sigma, 2007.

Elsholz, Uwe. Gewerkschaftliche Netzwerke zur Kompetenzentwicklung. Qualitative Analyse und theoretische Fundierung als Lern- und Organisationsform. München/Mering: Rainer Hampp, 2006.

Elsholz, Uwe; Knutzen, Sönke. "Lernortkooperation im Kopf durch E-Portfolios in der Ausbildung.» Berufsarbeit von morgen in gewerblich-technischen Domänen. Forschungsansätze und Ausbildungskonzepte für die berufliche Bildung. Hrsg. v. Claudia Fenzl, Georg Spöttl, Falk Howe und Matthias Becker. Bielefeld: Bertelsmann, 2009. 94-99.

Elsholz, Uwe; Pross, Gerald. «Arbeiten und Lernen strukturiert verbinden. Arbeitsintegrierte Fortbildung in der Entsorgungswirtschaft.» BWP - Berufsbildung in Wissenschaft und Praxis 37.2 (2008): 31-34.

Endres, Wolfgang; Wiedenhorn, Thomas; Engel, Anja (Hrsg.). Das Portfolio in der Unterrichtspraxis: Präsentations-, Lernweg- und Bewerbungsportfolio. Weinheim: Beltz, 2008.

Euler, Dieter (Hrsg.). Handbuch der Lernortkooperation. Band 1: Theoretische Fundierungen. Bielefeld: Bertelsmann, 2003.

Euler, Dieter. "Unter Weisskittel- und Blaukittelforschern: Aufgaben und Stellenwert der Berufsbildungsforschung.» Neue Forschungsverständnisse in den Sozialwissenschaften: Konsequenzen für die Berufsbildungsforschung im Bundesinstitut für Berufsbildung. Hrsg von Dieter Euler u. a. (2007) 43-74: www.bibb.de/dokumente/pdf/233_wd_94_neue_forschungsverstaendnisse_ sozialwissenschaften.pdf (23.2.2010).

Gehler, Bianka. «Das Paderborner Portfolio Lehramt - eine phasenübergreifende Perspektive.» Portfolio - Kompetenzen - Standards. Neue Wege in der Lehrerbildung für berufsbildende Schulen. Hrsg. v. Eva M. Hertle und Peter F. Sloane. Paderborn: Eusl, 2007. 63-79. 
Gläser-Zikuda, Michaela; Hascher, Tina (Hrsg.). Lernprozesse dokumentieren, reflektieren und beurteilen. Lerntagebuch und Portfolio in Bildungsforschung und Bildungspraxis. Bad Heilbrunn: Klinkhardt, 2007.

Häcker, Thomas. «Portfolio als Instrument der Kompetenzdarstellung und reflexiven Lernprozesssteuerung.»bwp@ Berufs- und Wirtschaftspädagogik online, 8 (2005): www.bwpat.de/ausgabe8/haecker_bwpat8.pdf (23.2.2010).

Häcker, Thomas. Portfolio: ein Entwicklungsinstrument für selbstbestimmtes Lernen. 2. Aufl. Baltmannsweiler: Schneider-Verlag Hohengehren, 2007a.

Häcker, Thomas. «Portfolio - ein Medium im Spannungsfeld zwischen Optimierung und Humanisierung des Lernens.» Lernprozesse dokumentieren, reflektieren und beurteilen. Lerntagebuch und Portfolio in Bildungsforschung und Bildungspraxis. Hrsg. v. Michaela Gläser-Zikuda und Tina Hascher. Bad Heilbrunn: Klinkhardt, 2007b, 63-85.

Häcker, Thomas; Winter, Felix. «Portfolio - nicht um jeden Preis! Bedingungen und Voraussetzungen der Portfolioarbeit in der Lehrerbildung.» Das Handbuch Portfolioarbeit. Hrsg. v. Ilse Brunner, Thomas Häcker und Felix Winter. 2. Aufl. Seelze-Velber: Kallmeyer, 2008. 227-233.

Hendrich, Wolfgang. «Beschäftigungsfähigkeit oder Berufsbiographische Gestaltungskompetenz.» Diskontinuierliche Erwerbsbiographien. Zur gesellschaftlichen Konstruktion und Bearbeitung eines normalen Phänomens. Hrsg. v. Friederike Behringer u. a. Baltmannsweiler: Schneider-Verl. Hohengehren, 2004. 260-270.

Hertle, Eva M. «Portfolio - ein kritischer Blick auf ein hoffnungsvolles didaktisches Instrument.» Lernen in medienbasierten kooperativen Lernumgebungen - Modellversuch KooL. Hrsg. v. H.-Hugo Kremer. Paderborn: Eusl, 2007. 121-138.

Hornung-Prähauser, Veronika; Geser, Guntram; Hilzensauer, Wolf; Schaffert, Sandra. «Didaktische, organisatorische und technologische Grundlagen von E-Portfolios und Analyse internationaler Beispiele und Erfahrungen mit E-PortfolioImplementierungen an Hochschulen.» (2007) www.fnm-austria.at/ePortfolio/ Dateiablage/view/fnm-austria_ePortfolio_Studie_SRFG.pdf (20.6.2009).

Howe, Falk; Berben, Thomas. "Lern- und Arbeitsaufgaben.» Handbuch Berufsbildungsforschung. Hrsg. v. Felix Rauner. 2., aktualisierte Aufl. Bielefeld: Bertelsmann, 2006. 383-390.

Howe, Falk; Knutzen, Sönke. Die Kompetenzwerkst@tt - Ein berufswissenschaftliches E-Learning-Konzept. Göttingen: Cuvillier, 2007.

Hübner, Sandra; Nückles, Matthias; Renkl, Alexander. «Lerntagebücher als Medium selbstgesteuerten Lernens - Wie viel instruktionale Unterstützung ist sinnvoll.» Empirische Pädagogik 21.2 (2007): 119-137.

Knutzen, Sönke; Howe, Falk. «E-Learning im Handwerk.» Online-Lernen. Handbuch für Wissenschaft und Praxis. Hrsg. v. Ludwig Issing und Paul Klimsa. München: Oldenbourg, 2009. 439-446. 
Lave, Jean; Wenger, Etienne. Situated learning. Legitimate peripheral participation. Cambridge: University Press, 1991.

Mandl, Heinz; Gruber, Hans; Renkl, Alexander. «Situiertes Lernen in multimedialen Lernumgebungen.» Information und Lernen mit Multimedia und Internet. Hrsg. v. Ludwig Issing und Paul Klimsa. Weinheim: Beltz, 2002. 139-148.

Ness, Harry. «Portfolioarbeit zur Anerkennung informell erworbener Kompetenzen in der Lehrerbildung.» Bildungsforschung.org 6.1 (2009): www.bildungsforschung.org/Archiv/2009-01/Portfolio/ (23.2.2010).

Payome, Thea. «Marktübersicht Rapid E-Learning - aus Powerpoint-Folien werden Lernprogramme.» Handbuch E-Learning - Expertenwissen aus Wissenschaft und Praxis. Hrsg. v. Karl Wilbers und Andreas Hohenstein, Köln: Deutscher Wirtschaftsdienst, 2008.

Schaffert, Sandra; Hornung-Prähauser, Veronika; Hilzensauer, Wolf; WiedenBischof, Diana. «E-Portfolio-Einsatz an Hochschulen: Möglichkeiten und Herausforderungen.» 〈 $\mathrm{Ne}(\mathrm{x}) \mathrm{t}$ Generation Learning〉: E-Assessment und EPortfolio: Halten sie, was sie versprechen? Hrsg. v. Taiga Brahm und Sabine Seufert (2007) St. Gallen: 74-89. www.salzburgresearch.at/research/ gfx/brahm-seufert-next-generation-learning.pdf (23.2.2010).

Schmidt, Joachim. «Portfolioarbeit an beruflichen Schulen. Perspektiven für Unterricht und Lehrerbildung» (2005): www.kibor-tuebingen.de/fileadmin/ user_upload/ibor_upload/pdf/Artikel___Portfolioarbeit_an_BS-korrigiert1.pdf (23.2.2010).

Seufert, Sabine; Brahm, Taiga. «E-Assessment und E-Portfolio zur Kompetenzentwicklung: neue Potenziale für $\mathrm{Ne}(\mathrm{x}) t$ Generation Learning.» ‘ $\mathrm{Ne}(\mathrm{x}) t$ Generation Learning: E-Assessment und E-Portfolio: Halten sie, was sie versprechen? Hrsg. v. Taiga Brahm und Sabine Seufert (2007): St. Gallen: 2-26. www.scil.ch/ fileadmin/Container/Leistungen/Veroeffentlichungen/2007-03-brahm-seufertnext-generation-learning.pdf (23.2.2010).

Witt, Claudia de; Czerwionka, Thomas. Mediendidaktik. Bielefeld: Bertelsmann, 2007.

Zeder, Andrea. Das Lernjournal: ein Instrument zur Förderung metakognitiver und fachlicher Kompetenzen. Paderborn: Eusl, 2006.

Weitere Informationen zum Projekt «Kompetenzwerkst@tt Elektrohandwerk»sowie eine Umsetzung des E-Portfolios sind zu finden unter http://www.kompetenzwerkstatt.net/e-handwerk 